\title{
Fallbeispiel H-Net - ein schweizerischer Intermediär im Gesundheitswesen
}

Der Beitrag führt kurz in das Thema der Intermediation im Gesundheitswesen ein und illustriert entsprechende Wertschöpfungspotenziale. Danach wird ein Fallbeispiel anhand der H-Net AG aus der Schweiz gegeben, an dem folgende Sachverhalte dargestellt werden: über H-Net verbundene Partner, von H-Net angebotene Plattform und Technologie sowie eine kurze Darstellung betriebswirtschaftlicher, medizinischer und technischer Dienstleistungen. Ferner erfolgt ein Preisvergleich des elektronischen Rechnungsdatenaustauschs mit den zwei direkten Konkurrenten Medidata und Trustcenter.

\section{Inhaltsübersicht}

1 Einleitung

2 Fallstudie $\mathrm{H}$-Net AG

2.1 Firmenportrait

2.2 H-Net ${ }^{\circledR}$-Plattform und darüber verbundene Partner im Gesundheitswesen

2.3 "H-Net ${ }^{\circledR}$-Business-Connector"

2.4 Prozessabwicklung ohne und mit $\mathrm{H}$-Net-Einsatz

2.5 Pricing der Dienstleistungen im Vergleich zu den Wettbewerbern

2.6 Einsparpotenziale durch elektronische Leistungsverrechnung

3 Zusammenfassung und Ausblick

4 Literatur

\section{Einleitung}

Die Bedeutung des elektronischen Geschäftsverkehrs (EGV) nimmt im Gesundheitswesen der Schweiz in den letzten Jahren massiv zu. Dies hat nicht zuletzt mit der Kostensituation im schweizerischen Gesundheitswesen zu tun, dessen Gesamtkosten derzeit 50 Mrd. CHF überschreiten [Bundesamt für Statistik 2003]. Mit dem EGV lassen sich Transaktionskosten zwischen den Beteiligten senken, da das Gesundheitswesen durch einen hohen Anteil vernetzter administrativer, medizinischer und logistischer Tätigkeiten und Kommunikationsbeziehungen mit vielen Akteuren gekennzeichnet ist.

Zur Beziehungskomplexität im schweizerischen Gesundheitswesen und zur Bedeutung des EGV trägt u.a. die Anzahl Akteure bei. So existieren im schweizerischen Gesundheitswesen ca. 700 Krankenhäuser und ähnliche Institutionen, ca. 19.000 Ärzte, 1.900 Apotheken, 300 Labore und 100 Versicherer oder Kostenträger, die administrative oder medizinische Datenaustausche haben können. Zudem kann davon ausgegangen werden, dass für alle diese Akteure unterschiedliche Softwarelösungen für die Verrechnung vorhanden sind. Ausgehend von der Vielfalt der zu vernetzenden Unternehmen und deren möglichen Informationssystemen befasst sich der vorliegende Beitrag mit der Analyse eines Intermediärs-Geschäftsmodells, der schweizerischen H-Net AG.

Unter dem Begriff des Intermediärs werden unabhängige, ökonomisch handelnde »Agenten « mit der Aufgabe verstanden, Transaktionen zwischen Wirtschaftssubjekten möglichst kostengünstig zu gestalten. Traditionelle Intermediäre besitzen Kernkompetenzen auf dem Weg der Verteilung eines Produktes oder einer Dienstleistung vom Hersteller zum Kunden. Hierzu zählen z.B. die zeitliche, räumliche oder finanzielle Überbrückung zwischen Partnern, der Güterausgleich, Beratungs- und Informationsleistungen oder aber der Datenaustausch 
zwischen den Partnern [Hartert 2002, S. 27; Brenner \& Schubert 1998, S. 183 f.]. Durch die Intermediation resultiert typischerweise eine Reduktion der Transaktionskosten, da die Beteiligten in einem Transaktionsraum "nur noch einmal« mit dem Intermediär statt mit unzähligen möglichen Transaktionspartnern in Kontakt zu treten haben.

Der in diesem Beitrag mehrfach erwähnte TARMED (»Tarif Médical«) bezeichnet den medizinischen Tarif, über den Leistungserbringer (Ärzte) ihre ambulanten Dienstleistungen verrechnen können (inklusive ambulanter Leistungen in Krankenhäusern). Der TARMED stellt sicher, dass gleiche ärztliche Dienstleistungen "schweizweit zur gleichen Anzahl Taxpunkte" ver- und abgerechnet werden [Aerosana 2004, S. 4]. Der Taxpunktwert wird kantonal festgelegt. Für die Verrechnung stationärer Leistungen zwischen Krankenhäusern und den Kostenträgern gelangen bis auf Weiteres kantonale Tarife zur Verrechnung. Diese sollen ab ca. 2009 durch den einheitlichen DRG (Diagnose-orientierte Leistungsverrechnung oder Diagnosis Related Groups) ersetzt werden [Fischer 2004], der in enger Anlehnung an das deutsche Verrechnungssystem entwickelt und implementiert wird.

Im schweizerischen Gesundheitswesen existieren zwei unterschiedliche Abrechnungsverfahren für Dienstleistungen im Gesundheitswesen. Es sind dies das Tiers-Payant- und das Tiers-Garant-Verfahren [Walser \& Egle 2005]. H-Net ${ }^{\circledR}$ unterstützt beide Abrechnungsverfahren. Im Tiers-Garant-Verfahren erhält der Patient die Rechnung vom Leistungserbringer direkt und rechnet danach mit der Versicherung ab. Im Tiers-Payant-Verfahren rechnet der Leistungserbringer mit dem Kostenträger $a b$ und dieser kassiert, falls erforderlich, den Rechnungsbeitrag oder entsprechende Differenzen vom Patienten.

In diesem Beitrag werden folgende Themenbereiche anhand des Intermediärs $\mathrm{H}$-Net beleuchtet: über den Intermediär verbundene
Partner, Träger- oder Eigentumsverhältnisse [Zbornik 1996, S. 127; Hartert 2002, S. 68], angebotene Dienstleistungen betriebswirtschaftlicher und/oder technischer Natur, betriebswirtschaftliche und technische Überlegungen des Intermediärs in Relation zu den durch ihn verbundenen Partnern (betriebswirtschaftliche Topologien: [Zbornik 1996, S. 127], [Hartert 2002, S. 68]; technische Topologien: [Kaib 2004, S. $53 \mathrm{ff}]$.$) , Kategorisierung von Leistungsange-$ bot und Intermediärsfunktion [Bailey 1998], Kosten-Nutzen-Verhältnisse der Intermediärsdienstleistungen, Marktabdeckung sowie Marktanteile im Marktsegment zwischen Kostenträgern und Leistungserbringern.

\section{Fallstudie H-Net AG}

\subsection{Firmenportrait}

2001 entstand die Integic, die aus der Seebeyond Schweiz (Produktevertrieb der Enterprise-Application-Integration-Suite Seebeyond) hervorging und u.a. auf die Integration im Krankenhausbereich spezialisiert ist. Zu den Kunden von Integic zählen u.a. Finanzdienstleister und zahlreiche staatliche und private Krankenhäuser. Die Integic ist tätig im Vertrieb und der Implementierung der Sun-Seebeyond-Lösung. Nach 2001 regten zunehmend Krankenhäuser eine neue Lösung für den Electronic Data Interchange (EDI) an. Ab 2003 wurde mit der erwähnten Integrationsplattform das Transaction-Delivery-Network-(TDN-)Konzept $\quad \mathrm{H}-\mathrm{Net}^{\circledR}$ zwischen drei Krankenhäusern erprobt und im selben Jahr ist die Firma H-Net AG als Betreiberin der Plattform gegründet worden. Die $\mathrm{H}$-Net AG ist ein neutraler Partner und finanziell mit keiner Partei oder politischen Kraft im Gesundheitswesen der Schweiz verflochten [Böni 2005]. Die Eigner der Firma sind die Mitarbeiter. $\mathrm{Zu}$ den direkten Konkurrenten von H-Net zwischen Krankenhäusern, Ärzten und Kostenträgern gehören die Medidata AG und die Trustcenter zusammen mit dem Health Info Net (HIN). 


\subsection{H-Net ${ }^{\circledR}$-Plattform und darüber verbundene Partner im Gesundheitswesen}

Die $\mathrm{H}$-Net ${ }^{\circledR}$-Plattform bietet aus technischer Sicht ein Netzwerk auf der Basis von Sun-Seebeyond und deren Produkt e*Gate Integrator an, das in der Schweiz zunächst innerhalb von Krankenhäusern zur Integration der verschiedenen IT-Systeme eingesetzt wurde. Ab 2004 realisierte die $\mathrm{H}-\mathrm{Net} \mathrm{AG}$ den produktiven Betrieb der eFaktura; Letzteres ist heute ein Teilmodul der Intermediationslösung von H-Net. Ab den Jahren 2005 und 2006 lassen sich immer mehr Partner im Gesundheitswesen über die H-Net ${ }^{\circledR}$-Plattform verbinden. Zunächst wurde im Krankenhausbereich von insgesamt 1,5 Mio. Rechnungen pro Jahr ausgegangen, die von Leistungserbringern zu Kostenträgern ausgetauscht werden. Heute sind es bereits 2 Mio. Rechnungen im Krankenhausbereich, die über $\mathrm{H}$-Net ${ }^{\circledR}$ übermittelt werden. Je nach Kanton variieren die elektronischen Verarbeitungen der Rechnungen zudem zwischen $20 \%$ und $80 \%$. Es gibt Kantone, die (aus politischen oder Kosteneinsparungsgründen) mehr Wert auf die elektronische Verrechnung legen als andere. Limitierend wirkt bei den Versicherern oder Kostenträgern die mangelhafte Integration der Informationssysteme, die für die elektronische Verrechnung relevant sind.

Das für die Plattform eingesetzte Produkt $\mathrm{e}^{*}$ Gate Integrator von Sun-Seebeyond ist busorientiert aufgebaut [Böni 2005]. Es handelt sich dabei um die Produktesuite $e^{*}$ Gate 4.5.x. Darauf basiert auch der eigentliche Kern von $\mathrm{H}$-Net ${ }^{\circledR}$, einer $\mathrm{n}: \mathrm{m}$-Hub-and-Spoke-Infrastruktur [Kaib 2004, 85 ff.; 135 ff.; Linthicum 2000; Winkeler et al. 2001, 7 ff.]. Der Hub stellt ein internetbasiertes Transaction Delivery Network (TDN) dar (vgl. zum Begriff Transaction Delivery Network [Altman 2001]). Es werden fachspezifische Meldungstypen in unterschiedlichen Datenformaten ausgetauscht, etwa: Leistungsabrechnungen, Apothekerrechnungen, Laborauf- träge und -befunde, medizinische Befunde, radiologische Bilder, Materialbestellungen sowie Zahlungsaufträge und -eingänge. Es erfolgt eine Systemintegration des $\mathrm{H}$-Net-Anschlusses in die Infrastruktur der Nutzer von H-Net. Die Statusverwaltung der Messages oder Datenaustausche erfolgt $\mathrm{H}$-Net ${ }^{\circledR}$-zentral. Es werden keine Daten gehalten, sondern nur solche weitergegeben.

Die $\mathrm{H}$-Net ${ }^{\circledR}$-Plattform bietet maximale Sicherheitsstandards, d. h. SSL-2048-Bit-Verschlüsselung, an. Dies stellt eine notwendige Voraussetzung im Gesundheitswesen dar. H-Net ${ }^{\circledR}$ ermöglicht einen revisionstauglichen Datenaustausch sowie die freie Wahl der Datenformate auf der Sender- und Empfängerseite. Es erfolgen durch die $\mathrm{H}$-Net ${ }^{\circledR}$-Plattform eine formale Validierung und ein automatisches Errorhandling, auch ein ereignisgesteuertes Routing ist möglich. Die Plattform ist fehlertolerant. Daten, Bild und Ton können in beliebigen Größen übertragen werden. Dies ist im medizinischen Bereich etwa für Computertomographie-Studien mit z.B. 3.000 Bildern von hohem Wert. Der Anschluss ist einfach und flexibel handhabbar. Die Plattform ist sehr leistungsfähig und ermöglicht einen wirtschaftlichen Datenaustausch.

Die H-Net ${ }^{\circledR}$-Plattform wird gezielt von einer (technischen) Datenintegrationsplattform zur umfassenden (betriebswirtschaftlichen und medizinischen) Integrationsplattform im Gesundheitswesen entwickelt. Es können faktisch alle wünschbaren Datenformate über die $\mathrm{H}$ Net ${ }^{\circledR}$-Plattform integriert und abgewickelt werden. Das Ansinnen des Datenforums Schweiz, über XML-Standards die Formate verschiedener Verrechnungsgeschäftsvorfälle zu standardisieren, ist aufgrund der Diversität der beteiligten Informationssysteme bei Leistungserbringern und Kostenträgern kurz- und mittelfristig unrealistisch. $\mathrm{H}$-Net setzt ausgehend von seinem Integrationsverständnis auf die Datentransformation und nicht nur auf die thematisierte Standardisierung. Jedoch werden die entsprechenden Standards auch von H-Net unterstützt. 
Eine Reduktion der Transaktionskosten resultiert für Kunden der $\mathrm{H}$-Net ${ }^{\circledR}$-Plattform dadurch, dass fast alle Datenformate für ein- und ausgehende Meldungen möglich sind und dafür entsprechende Transformationen angeboten oder in sehr kurzer Zeit neu implementiert werden. Die H-Net nimmt den entsprechenden Leistungspartnern das Datensourcing, die Datentransformation, allenfalls das Splitting der Daten sowie das Laden ins gegenüberliegende System ab. Sind beispielsweise bei Krankenversicherern unterschiedliche Filialmodelle vorhanden, die den Rechnungsdatenaustausch betreffen, ist auch eine Verteilung zu Händen dezentraler Versicherungsstellen mittels Regelmechanismen in der $\mathrm{H}$-Net ${ }^{\circledR}$-Plattform realisierbar. Zusätzlich kompliziert wird die Punktzu-Punkt-Integration ohne Intermediär auch durch unterschiedliche Informationssystemtypen bei Krankenhäusern, Ärzten und Versicherern (d.h. der Anzahl Partner) oder innerhalb der Leistungserbringer, z. B. im Rahmen der Übermittlung von Röntgenbildern. Zu Letzterem bietet $\mathrm{H}$-Net eine voll integrierte Lösung ohne Medienbrüche auf Basis des Internetpro- tokolls für PACS (Picture Archiving and Communication System (für Digital Imaging and Communications in Medicine (DICOM) und auch non-DICOM)) an.

Die zwischenbetriebliche Integration über die $\mathrm{H}$-Net ${ }^{\circledR}$-Plattform wird entsprechend der Abbildung 1 zwischen folgenden Partnern ermöglicht [Böni 2005]: Krankenhäuser (aktuell ca. 130; zum Vergleich, Medidata bedient derzeit etwa 100, die EDS (Electronic Data Systems Corporation) etwa 13 Krankenhäuser), Ärzte (derzeit ca. 700; zum Vergleich, Medidata bedient derzeit ca. 1.000 Ärzte; die Trustcenter eine wohl fünfstellige Anzahl von Ärzten), eine Abrechnungskasse für Ärzte, eine Apotheke, drei Labore, sieben Radiologiestationen, ein Print Center, PostFinance, die Zürcher Kantonalbank (ZKB) und Paynet für EBPP (Electronic Bill Presentation and Payment), Logistikdienstleister wie etwa die Schweizerische Post oder Logigate, die Transaktionsplattform der Firma Global Healthcare Exchange (GHX), drei Trustcenter, 34 Versicherer über Mediport (Medidata-Roaming) und 44 Versicherer teilweise direkt.

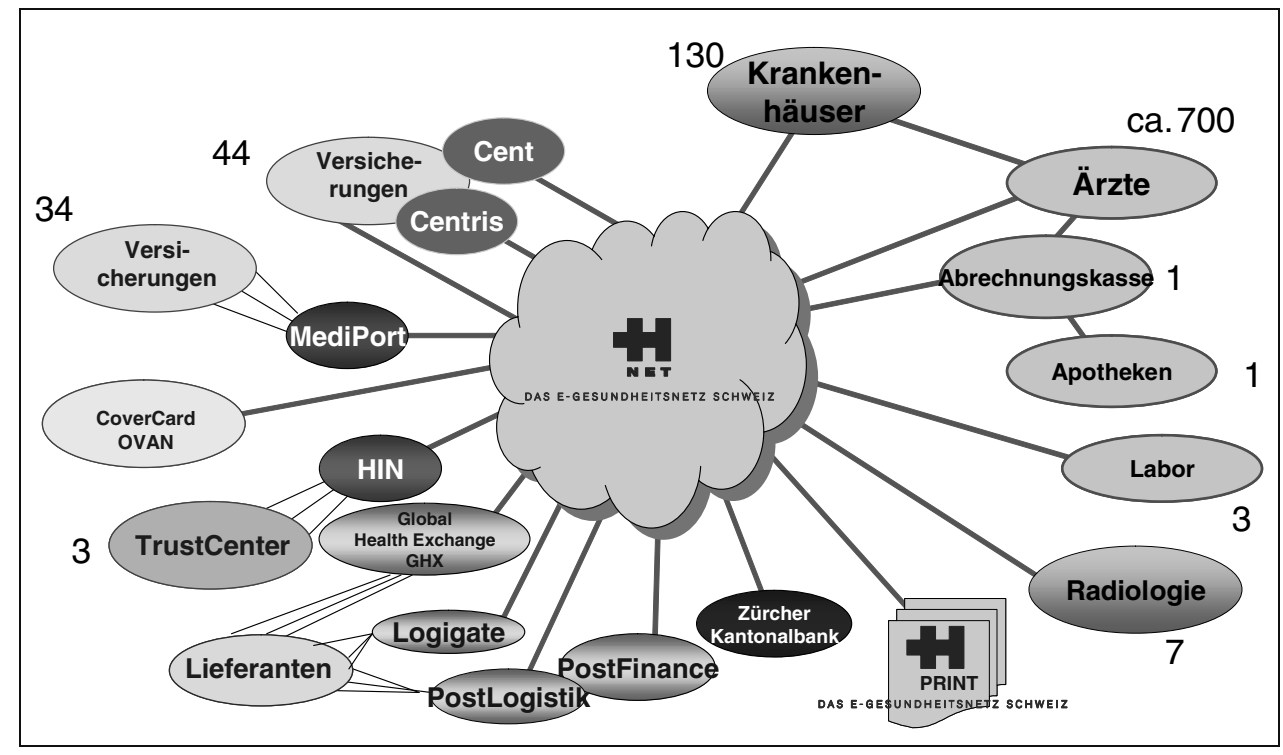

Abb. 1: Über H-Net ${ }^{\circledR}$ verbundene Partner [Böni 2005] 
In Abbildung 1 steht OVAN für OFAC Value Added Network. Die OFAC ist die Berufsgenossenschaft der Apotheker und zudem die Intermediärin zwischen Apothekern und Versicherern für den (Rechnungs-)Datenaustausch (Rezepte etc.). HIN steht für Health Info Net, einen technischen Intermediär im Gesundheitswesen der Schweiz. PostFinance ist der Finanzdienstleister der Schweizerischen Post. Die Paynet gehört zur Telekurs-Gruppe in Zürich, dem größten schweizerischen Intermediär im Finanzdienstleistungsbereich. PostFinance und Paynet bieten neben der Zürcher Kantonalbank EBPPDienstleistungen (elektronische Rechnungsstellung und -bezahlung) an. Über Paynet, die Tochter der Telekurs AG (Interbank-Clearing), sind fast alle Finanzdienstleister der Schweiz in das EBPP-Netzwerk integriert.

Die H-Net AG geht basierend von ihrer Intermediärsplattform von einer zweistufigen Geschäftsentwicklung aus. Auf einer ersten Stufe wurde die Integration im administrativen Bereich für Krankenhäuser verwirklicht (betriebswirtschaftliche Ebene des Datenaustausches, Rechnungsdatenaustausch). In einer zweiten Phase (Beginn ca. ab Anfang 2006) wird auf das Datenclearing im medizinischen Bereich zwischen den Leistungserbringern sowie gegenüber Beschaffungspartnern gesetzt. Dazu gehört z. B. der erwähnte Austausch von Radiologiedaten und -bildern oder anderen medizinischen Informationen.

\section{3 "H-Net ${ }^{\circledR}$-Business-Connector"}

Der H-Net ${ }^{\circledR}$-Business-Connector stellt das umfassende Interface oder den Adapter für die mit der $\mathrm{H}$-Net ${ }^{\circledR}$-Plattform verbundenen Partner dar. Ein nicht vollständiger Katalog von Intermediärsdienstleistungen, die über den BusinessConnector 4.0 angeboten werden, lautet wie folgt: eFaktura Tiers Payant, eFaktura Tiers Garant, Druck und Versand von Papierrechnungen, eProcurement und Delivery über PostLogistik, eProcurement via GHX/e-mediat, ePayment Post und Bank, Covercard-Abfrage sowie EBPP im $\mathrm{B}_{2} \mathrm{C}$-Bereich. Künftig sollen auch Kostengutsprachen für Krankenhäuser sowie Master-Patient-Index als Dienstleistungen ermöglicht werden. Unter anderen werden heute oder künftig folgende weitere Dienstleistungen erbracht: eXRay, eLab-Auftrag und eLab-Befund [Böni 2005]. Die weiter oben erwähnte Covercard ist ein Magnetkartenangebot der OFAC, die neben der Bereitstellung anderer Dienstleistungen die Rechnungsdaten der Apotheken bündelt oder Kostengutsprachen ermöglicht. So haben z.B. Krankenhäuser, Ärztinnen und Ärzte die Möglichkeit, administrative Daten ihrer Patienten via Browser oder direkt mit der Praxissoftware online abzurufen. Der Kartenstandard von OFAC wird auch von HIN (Health Info Net), einem anderen Intermediär, unterstützt. Zudem hat die santésuisse (Branchenverband der schweizerischen Krankenversicherer) im Herbst 2005 (zusammen mit der OFAC) beschlossen, dass die Covercard der aktuelle Standard für alle Versichertenkarten und die Grundlage für eine künftige Gesundheitskarte wird [santésuisse 2005].

Das Basispaket des $\mathrm{H}$-Net ${ }^{\circledR}$-Business-Connectors 4.0 mit den geschilderten Dienstleistungen basiert auf verschiedenen Modulen, die wie folgt lauten [Böni 2005]: Modul eFaktura ambulant (auf Basis des $\mathrm{H}$-Net ${ }^{\circledR}$-Secure-Clients); Modul eFaktura Tiers Payant ambulant, Modul eFaktura Tiers Garant ambulant, Modul Covercard-System, Modul ePayment PostFinance, Modul eProcurement PostLogistik und GHX, Modul EBPP mit Yellobill oder Paynet, Modul elektronische Kostengutsprache (ab 4. Quartal 2006; eKOGU) sowie das Modul XMIT/Rechnungsbeilagen. Kostengutsprachen sind Abklärungen von Krankenhäusern bei Krankenversicherern vor einer Behandlung eines Patienten, um Versicherungsstatus und -deckung des Patienten abzuklären. Bei XMIT handelt es sich um einen Standard für Data- oder Meldungs-Container. Mittels XMIT können Rechnungszusatzdaten im XML-Format versendet werden [Schellenberg 2005b]. Abbildung 2 zeigt die bereits erwähnten 


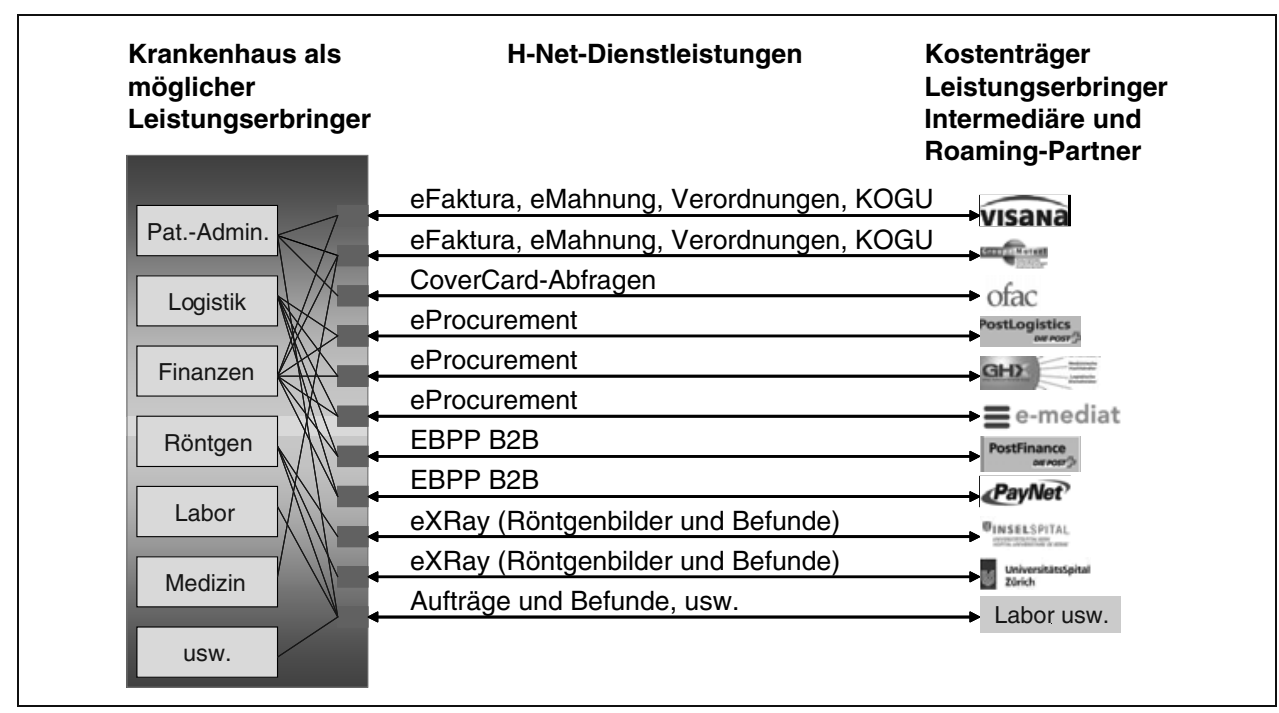

Abb. 2: Mögliche Produkte auf Basis des H-Net ${ }^{\circledR}$-Business-Connectors zwischen Versicherung und Partnern [Böni 2005]

Produkte in der Anwendung zwischen Krankenhaus und verschiedenen Partnern sowie im Bereich EBPP.

Damit die betriebswirtschaftlichen und medizinischen Dienstleistungen mittels elektronischen Geschäftsverkehrs oder elektronischer Prozessunterstützung von der H-Net AG angeboten werden können, sind noch verschiedene technische Anforderungen an die $e^{*}$ Gate-Plattform zu nennen: Chiffrierung/Dechiffrierung, Meldungssignatur, Transport mit H-Net ${ }^{\circledR}$-proprietärem Protokoll, garantierte Ein-/Auslieferung der Daten, Datenkomprimierung (z. B. für Radiologiedaten/DICOM), Formatkonvertierungen, ereignisbasiertes Splitting und/oder Routing, formale Validierung, automatisches Errorhandling, Transaction Monitoring, Journalisieren und Alarmfunktionen sowie das Angebot zur Implementierung von Web-Services. Es werden als Dienstleistungen ein technischer Support 7×24 Stunden und ein Helpdesk sowie zusätzlich zu den in Abbildung 2 dargestellten Dienstleistungen auch noch Data-ContainerFunktionalität (XMIT) angeboten.

\subsection{Prozessabwicklung ohne und mit H-Net-Einsatz}

Für die weitere Entwicklung wichtig dürfte das transparente Preis-Leistungs-Verhältnis und die Bereitschaft und Fähigkeit von $\mathrm{H}$-Net sein, sich rasch an die wandelnden Bedürfnisse der verbundenen Partner im Gesundheitswesen und entsprechende (gesetzliche oder Branchen-) Änderungen anzupassen. Eine vereinfachte Darstellung einer nicht integriert abgewickelten eFaktura mag zeigen, wie aufwendig ein solcher Prozess wird.

Das Krankenhaus versendet eine eFaktura (XML300). Der Versicherer macht eine Rückweisung, da der Patient nicht bekannt ist. Das Krankenhaus klärt $a b$ und versendet die eFaktura erneut (XML300). Der Versicherer führt eine Rückweisung durch, da der Tarif falsch angewendet wurde. Das Krankenhaus storniert die Rechnung und erstellt eine neue eFaktura. Das Krankenhaus versendet die eFaktura in XML40o. Der Versicherer kann XML400 erst später verarbeiten oder ist dafür nicht bereit. Das Krankenhaus 
wartet so lange, bis die Verarbeitung möglich ist, oder erstellt eine Papierrechnung. XML 300 und XML 400 stellen XML-Formate für elektronische Rechnungsantworten und Mahnungsantworten dar. Durch dieses Vorgehen entstehen beim Leistungserbringer neben unterschiedlich hohen Übermittlungskosten (u.a. auch wegen Medienbrüchen) auch intern zusätzliche administrative Kosten.

Es sei deshalb auf den im Folgenden dargestellten Verlauf einer Transaktion mit $\mathrm{H}_{-} \mathrm{Net}^{{ }_{-}}{ }_{-}$ Einsatz hingewiesen. Das Krankenhaus erfasst den Patient mittels Covercard-Abfrage. Das Krankenhaus stellt eine Kostengutsprache-Anfrage (KOGU) elektronisch an die Versicherung. Das Krankenhaus erhält eine KOGU-Antwort elektronisch von der Versicherung. Das Krankenhaus erfasst Leistungen. Das Krankenhaus erstellt eine eFaktura (Format und Tarifkontrolle automatisiert). Das Krankenhaus versendet die eFaktura in beliebigem technischem Format. Die Versicherung erhält die eFaktura in einem für sie elektronisch lesbaren Format. Das Krankenhaus erhält einen Zahlungseingang direkt elektronisch z.B. über PostFinance und deren EBPP-Dienstleistung. Mit diesem Vorgehen können die Aufwands- und Administrationskosten im Vergleich zum vorher dargestellten Vorgehen gesenkt werden.

Auch für die Abwicklung des Kreditorengeschäfts bei Materialbestellungen auf der Beschaffungsseite der Leistungserbringer (z. B. für Krankenhäuser und Ärzte) ist die H-Net AG Kooperationen sowohl mit PostFinance, Paynet sowie der ZKB (Zürcher Kantonalbank) als auch mit GHX sowie der Logistikanbieterin "Die Schweizerische Post « eingegangen. Die H-Net AG positioniert sich mittels Roaming-Verträgen unter anderem und teilweise im Vergleich zu den direkten Konkurrenten Medidata und Trustcenter auch als Intermediär auf der Beschaffungsseite der Gesundheits-Wertschöpfungskette (zunächst für Krankenhäuser). Die Entwicklung der Nutzungszahlen ist positiv. Wesentlich für die künftige Entwicklung von
$\mathrm{H}$-Net dürften die Roaming-Politik, die Innovationskraft, die herausragenden technischen Fähigkeiten, die Flexibilität und der effiziente und ökonomische Umgang mit den (finanziellen und technischen) Ressourcen sein.

\subsection{Pricing der Dienstleistungen im Vergleich zu den Wettbewerbern}

Im direkten Vergleich mit drei Konkurrenten auf dem Gebiet des elektronischen Rechnungsdatenaustauschs (eFaktura) zwischen Leistungserbringern und Kostenträgern können folgende Aussagen zu den Preisen der Intermediärsdienstleistungen von $\mathrm{H}$-Net gemacht werden. Die erweiterte Basis dazu bilden [Schellenberg 2005a], [Schellenberg 2006] sowie Auskünfte eines Interviewpartners bei einem mittelgroßen Kostenträger (vgl. dazu auch [Walser \& Egle 2005]).

Trustcenter: Die Kostenträger, die die Rechnungen bei den ärzteseitigen Trustcentern abholen, zahlen im Tiers Garant eine Transaktionsgebühr von $1 \mathrm{CHF}$. Die Trustcenter verlangen für die ausgehenden Rechnungen offiziell 1,40 CHF. Inoffiziell wird von $1 \mathrm{CHF}$ gesprochen. Zusätzlich zu diesen Kosten fallen noch 0,02 bis 0,03 CHF an für die Übermittlung über die Plattform von HIN sowie eine monatliche Gebühr von 50 CHF pro Trustcenter, von dem Rechnungen bezogen werden. Werden beispielsweise von (allen) 11 Trustcentern Rechnungen bezogen, ergibt das einen monatlichen Betrag von $550 \mathrm{CHF}$.

Medidata: Für die Leistungserbringer ist die Einspeisung der Rechnungen wie erwähnt kostenlos. Die Kostenträger zahlen unterschiedliche Preise. Die großen fünf Kostenträger und Hauptaktionäre von Medidata zahlen Pauschalpreise im sechs- bis siebenstelligen Bereich pro Jahr (insgesamt rund 8,4 Mio. CHF gemäß [O.V. 2006]). Darin sind alle Dienstleistungen, die Medidata bietet, eingeschlossen, z.B. auch die Nutzung diverser Kataloge und entsprechende Datenzugriffe [Walser \& Egle 2005]. Die nicht zu den fünf großen schweizerischen Versicherern gehörenden Kostenträger haben Kosten von 
0,30 CHF bei Konzentratoren im Tiers Payant (z.B. OFAC) und o,9o CHF bei Kostenträgern im Tiers Garant pro Rechnung zu tragen. Der offiziell kommunizierte Preis liegt jedoch im Tiers Payant bei 1,40 CHF.

$H$-Net führt zwei Rechenmodelle für zwei unterschiedliche Dienstleistungen. Für den Business-Connector, der den größten Dienstleistungsumfang enthält [Böni 2005], fallen jährlich je nach Größe des Unternehmens tiefe bis hohe fünfstellige Kosten an. Für die Transaktion der eFaktura, eine Teildienstleistung des Business-Connectors, fallen Senderkosten von 0,25 bis 0,50 CHF (für den Arzt, respektive das Krankenhaus) an. Wird beispielsweise eine gestellte Rechnung vom Absender verschickt, zahlt er 0,50 CHF. Eine Rückweisung der Rechnung (Anteil kleiner als $10 \%$ ) durch den Kostenträger schlägt wieder mit 0,50 CHF zu Buche.

EDS (Nutzer der Krankenhaussoftware DIOHIS von EDS) verfolgt das gleiche Preismodell wie H-Net. Verursacher zahlen pro versendete Rechnung 0,50 CHF. An EDS angeschlossen sind ca. 13 Krankenhäuser. Über einmalige Anschluss- oder Pauschalgebühren ist nichts bekannt.

Es erfolgt heute erst mit circa sieben von 100 Krankenversicherern ein durchgängig integrierter elektronischer Datenaustausch. Bei einem von diesen, einem mittelgroßen Kostenträger, erfolgt erst eine vollständige elektronische Einspeisung und Verarbeitung für ca. $50 \%$ von etwa 3,5 Mio. Rechnungen jährlich. Ausgehend von den drei Finanzierungsarten der Intermediäre (durch Leistungserbringer, unabhängig, durch Kostenträger), aber auch abhängig von anderen Kriterien, lässt sich über unterschiedliche Erfolge der Intermediäre diskutieren.

\subsection{Einsparpotenziale durch elektronische Leistungsverrechnung}

Das Sparpotenzial, das sich aufgrund eines vereinbarungsgemäßen elektronischen Datenaustauschs zwischen Leistungserbringern (hier aus der Sicht des Krankenhauses) und -trägern nach
[Ziegler 2006] ergibt, lautet wie folgt: Der Papierweg der Verrechnung generiert im Krankenhaus Kosten von 4 bis 8 CHF. Darin eingeschlossen sind die Rechnungsaufbereitung, der Druck, die Verpackung und der Postversand. Bei den Versicherern resultieren Kosten der Papierrechnung von 8 bis 12 CHF. Darunter sind Prozesskosten zu subsumieren wie Scanning, Nachbearbeitung, Validierung und Einlesen in die versicherungseigene Software. Der elektronische Weg dagegen generiert bei den Krankhäusern bei der Übermittlung mit H-Net Kosten von 0,50 CHF und bei den Kassen von o CHF. Aus der volkswirtschaftlichen Perspektive ließen sich damit auf dem elektronischen Weg bei der Rechnungsabwicklung des Krankenhauses mindestens $10 \mathrm{CHF}$ einsparen. Nicht quantifiziert resultieren zusätzlich Zeit- und (Daten-)Qualitätsgewinne. Bei einer Schätzung von ca. 5 Mio. jährlichen Rechnungen von Krankenhäusern resultieren damit Einsparpotenziale von 50 Mio. CHF. Dieses Einsparpotenzial ist bei den Ärzten noch wesentlich größer, insbesondere aufgrund von Economies of Scale. [Schellenberg 2006] gibt Einsparpotenziale von mindestens 3,50 CHF für den Vergleich elektronisch verarbeitete versus herkömmliche Papierrechnung an. Die geringeren Einsparpotenziale ergeben sich bei den Leistungserbringern, die den Druck und Versand der Papierrechnung - je nachdem, ob Tiers Payant oder Tiers Garant zum Einsatz gelangt, unterschiedlich - vermindern können. Die größeren Einsparpotenziale liegen bei den Kostenträgern, die geringere Prozesskosten für das Einlesen und die Verarbeitung der Rechnungen haben.

\section{Zusammenfassung und Ausblick}

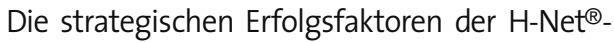
Plattform lauten wie folgt: Sie ermöglicht eine Einhaltung der TARMED-Vereinbarung zwischen Leistungserbringern und Kostenträgern. Sie bietet die technischen Grundlagen (e*GatePlattform von Sun-Seebeyond, H-Net ${ }^{\circledR}$-Trans- 
action Delivery Network) und Weiterentwicklungspotenziale, die damit einen Beitrag zur ökonomischen Gesundung des schweizerischen Gesundheitswesens hinsichtlich Zeit-, Kostenund Qualitätskriterien leisten können. Die $\mathrm{H}$ Net ${ }^{\circledR}$-Plattform bietet zudem eine breite Dienstleistungspalette im elektronischen Geschäftsverkehr über die gesamte Wertschöpfungskette des schweizerischen Gesundheitswesens an. Zu erwähnen ist ferner die Breite des unmittelbaren Portfolios an Intermediärs(dienst)leistungen auf betriebswirtschaftlicher und technischer Ebene. Das Unternehmen $\mathrm{H}$-Net ist finanziell unabhängig bezüglich Parteien im Gesundheitswesen, hat aber aus historischen Gründen eine gewisse Nähe zur Krankenhausbranche. H-Net hat zudem nachweisbare Fähigkeiten auch in anderen Branchen. Interessant für die weitere Entwicklung des Intermediärs sind dessen Netzwerkgröße und die Art der Anbieter und Kunden (Netzwerkeffekte etwa im Sinne Metcalfes Law [Shapiro \& Varian 1998]) sowie die Art und Anzahl der Roaming-Abkommen und -Partner. Wichtig für eine erfolgreiche Weiterentwicklung sind die Individualisierbarkeit der Dienstleistungspalette aus der Kunden- oder Partnerperspektive und der unabhängige Interessenausgleich zwischen den zusammenarbeitenden Partnern. Der Wettbewerb unter den Intermediären der Gesundheits-Wertschöpfungskette ist ein Wettbewerb bezüglich betriebswirtschaftlicher, medizinischer und technischer Services oder Dienstleistungen, die auf technischen Plattformen angeboten werden. Diesbezüglich ist $\mathrm{H}$-Net gut positioniert.

\section{Literatur}

[Aerosana 2004] Aerosana: Was ist der TarMed, 2004, www.aerosana.ch/pdf/de bulletin_1_04. pdf; Zugriff am 04.01.2005.

[Altman 2001] Altman, R.: What is a Transaction Delivery Network, 2001, http://gartner.lib. depaul.edu/gartner intraWeb/research/97900/ 97952/97952.pdf; Zugriff am 06.06.2006.
[Bailey 1998] Bailey, J. P.: Intermediation and Electronic Markets: Aggregation and Pricing in Internet Commerce, 1998, www.smith.umd.edu/ faculty/jbailey/pub/phdthesis.pdf; Zugriff am 30. 12.2004 .

[Böni 2005] Böni, S.: H-Net - Die Integrationsplattform für das Schweizer Gesundheitswesen. Präsentation vom 29.09.2005 vor der Swiss ICT Fachgruppe Investment, www.pascal-sieber.net/ files/cno/cno-05/fg12-stefan-boeni.pdf; Zugriff am 07.03.2006.

[Brenner \& Schubert 1998] Brenner, W.; Schubert, C.: Cyber-Intermediaries - Die neuen Dienstleister des 21. Jahrhunderts: Eine Rollen- und Wertschöpfungstheoretische Analyse. In: Burkhardt, T.; Lohmann, K. (Hrsg.): Banking und Electronic Commerce im Internet. Spitz, Berlin, 1998, S. 131-159.

[Bundesamt für Statisktik 2003] Bundesamt für Statistik - Gesundheitsobservatorium: Kosten des Gesundheitswesens (der Schweiz), 2003, www.bfs.admin.ch/bfs/portal/de/index/themen/ gesundheit/gesundheitsversorgung/kosten finan zierung/kennzahleno/kosteno/ueberblick.html; Zugriff am 14.06.2006.

[Fischer 2004] Fischer, W.: Gesucht: Ein DRG-System für die Schweiz - Kurzer Überblick zum Beginn der Arbeiten am SwissDRG-Projekt, 2004, www. swissdrg.org/assets/pdf/de/DRG-CH-Modellwahl0404.pdf; Zugriff am 06.12.2005.

[Hartert 2002] Hartert, D.: Global Virtual Market Konzept für einen dezentral organisierten elektronischen Markt, 2002, http://bibserv7.bib.unimannheim.de/madoc/volltexte/2003/59/pdf/59 1.pdf.

[Kaib 2004] Kaib, M.: Enterprise Application Integration - Grundlagen, Integrationsprodukte, Anwendungsbeispiele. DUV, Wiesbaden, 2004.

[Linthicum 2000] Linthicum, D.: Enterprise Application Integration. Addison-Wesley, Boston, 2000.

[O.V. 2006] o. V.: Versicherer bitten doppelt zur Kasse. In: Sonntagszeitung vom 06.03.2006, S. 68.

[santésuisse 2005] santésuisse: Das Versichertenkartencenter (VeKa) von santésuisse und Ofac bestätigen offiziell ihre Zusammenarbeit bei der europäischen Versichertenkarte, 2005, www.konsum.ch/kf-news/Communique santesuisse ofac D.pdf; Zugriff am 06.06.2006. 
[Schellenberg 2005a] Schellenberg, R.: Was darf eine elektronische Rechnung kosten? Mai 2005, www.portx.ch/de/media/files/klinik_und_heim_ 5-05_paper-sel.pdf; Zugriff am 01.03.2006.

[Schellenberg 2005b] Schellenberg, R.: Vor- und Nachteile zukunftsorientierter Lösungsansätze. Proceedings der eHealthcare 05 in Nottwil/CH, 2005, www.portx.ch/de/media/files/eHealthcare05-referat-schellenberg.pdf; Zugriff am 06.06. 2006.

[Schellenberg 2006] Schellenberg, R.: Ambulante Rechnungen in der Schweiz: Was darf eine elektronische Rechnung kosten? 2006, www.ebpp info.de/portal/forum/schellenberg; Zugriff am 01.03.2006.

[Shapiro \& Varian 1998] Shapiro, C.; Varian, H.: Information Rules: A Strategic Guide to Network Economy. Harvard Business School Press, Boston, Mass., 1998.

[Walser \& Egle 2005] Walser, K.; Egle, U.: Intermediation im schweizerischen Gesundheitswesen. Arbeitsbericht Nr. 163, Institut für Wirtschaftsinformatik, Universität Bern, Bern, 2005.

[Winkeler et al. 2001] Winkeler, T.; Raupbach, E.; Westphal, L.: Enterprise Application Integration als Pflicht vor der Business-Kür. In: Information Management \& Consulting 1 (2001) 16, S. 7-16.

[Zbornik 1996] Zbornik, S.: Elektronische Märkte, elektronische Hierarchien und elektronische
Netzwerke: Koordination des wirtschaftlichen Leistungsaustausches durch Mehrwertdienste auf der Basis von EDI und offenen Kommunikationssystemen, diskutiert am Beispiel der Elektronikindustrie. Konstanz, 1996.

[Ziegler 2006] Ziegler, M.: Nationale Gesundheitsinfrastruktur - Die zentrale Bedeutung einer geordneten eHealth-Vernetzung Schweiz, Vortrag, gehalten am SGMI-Jahreskongress (Schweizerische Gesellschaft für Medizin-Informatik SGMI) vom 02.05.2006 in Basel, 2006.

Dr. rer. oec. Konrad Walser

Universität Bern

Institut für Wirtschaftsinformatik

Engehaldenstr. 8

$\mathrm{CH}-3012$ Bern

Konrad.walser@gmail.com

www.im.iwi.unibe.ch

Dipl.-Ing. ETH Dipl.-Wirtsch.-Inform. Michael Ziegler Stefan Böni

$\mathrm{H}$-Net AG

Stampfenbachstr. 40

CH-8006 Zürich

michael.ziegler@h-net.ch

stefan.boeni@freesurf.ch

www.h-net.ch 